\title{
Neuraltherapie bei nicht vertebragenen Schulterbeschwerden
}

Antiinflammatorische, schmerzlindernde und kortisonfreie Be-

handlungsoption, die komplementär zu konservativen und operativen Therapien eingesetzt werden kann - Über Vasodilatation und Sympathikolyse wirkt die Neuraltherapie zudem regenerativ

Abb. 1 @ Karl Wesker; Aus: Schünke M, SchulUwe Günter te $E$, Schumacher U. Prometheus. Stuttgart: Thieme; 2011

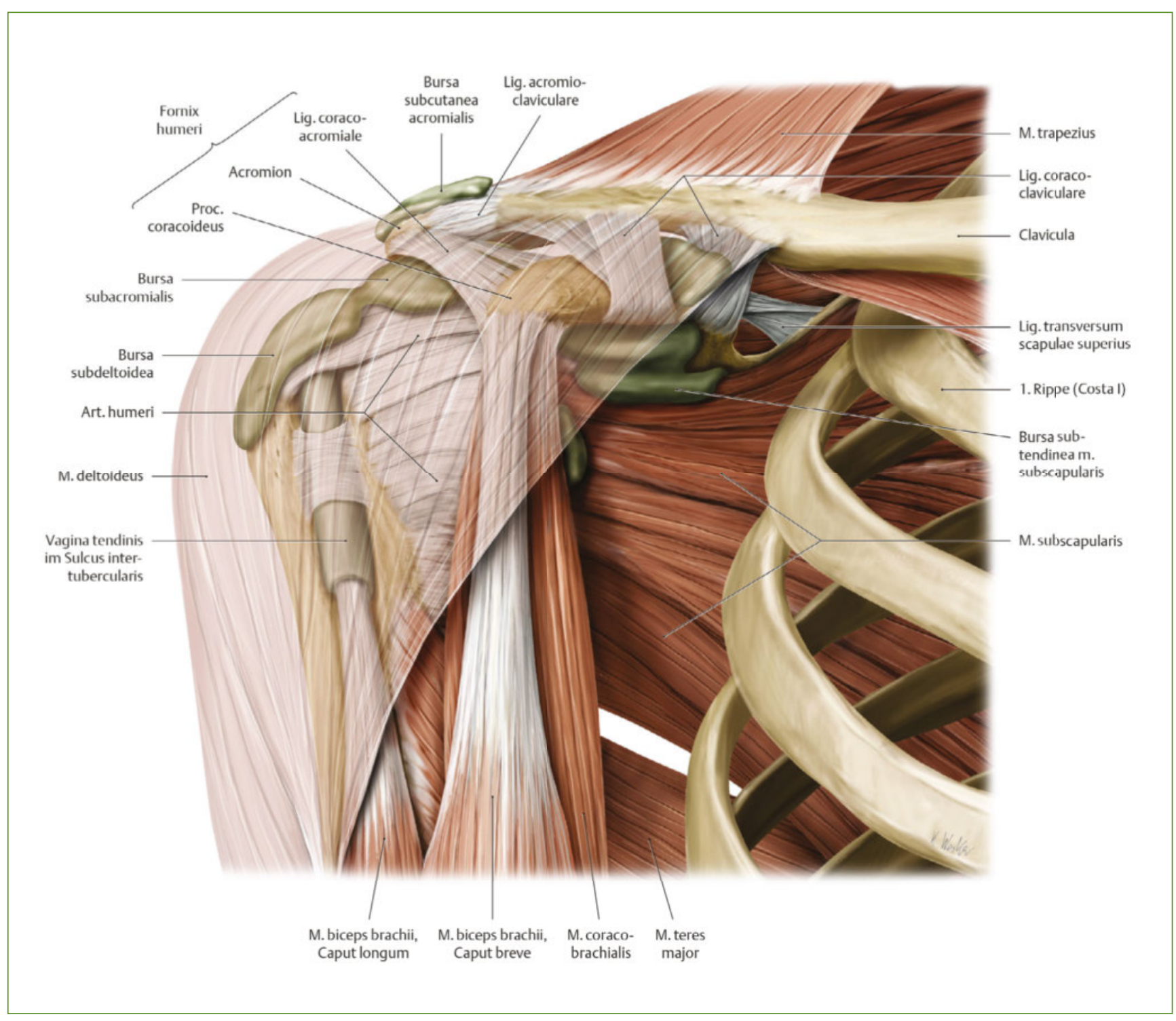




\section{Einleitung}

Schulterbeschwerden werden bei Ausstrahlung als Cervico-Omalgie bzw. Omo-Brachialgie bezeichnet. Die als Omalgie zusammengefassten Beschwerden werden je nach Klinik sehr variabel beschrieben. Parallel zu den klinischen Entitäten impingement, frozen shoulder, thoracic outlet und complex regional painsyndrome wird je nach segmentaler Projektion des Schmerzes auch nach den Dermatomen als C4-Schmerz (oberhalb der Clavicula), C5-Schmerz (im Bereich des Deltamuskels) usw. dokumentiert.

Aus neurovegetativer Sicht kommt dem perivaskulären Sympathikus eine bedeutende Rolle bei Schmerzen zu. Auch der Nervus phrenicus sowie andere Afferenzen müssen immer beachtet werden. Die Symptomatik zeigt sich mannigfaltig und bedarf einer individuellen manuellen Diagnostik.

\section{Ursache und Häufigkeiten von Omalgien}

Je nach Ursache, Häufigkeit und Differenzialdiagnose unterscheidet man angeborene und erworbene Pathologien. Nichttraumatische Veränderungen sind schwieriger zu unterscheiden.

Neben den angeborenen Pathologien einer Sprengel-Deformität, einer Halsrippe oder eines Akromionsporns stehen Unfälle und Überlastungen („over used“) durch Beruf und Sport in der Traumatologie und Sportmedizin im Vordergrund. Bekannte Präarthrosen wie Knorpelverletzungen tre-

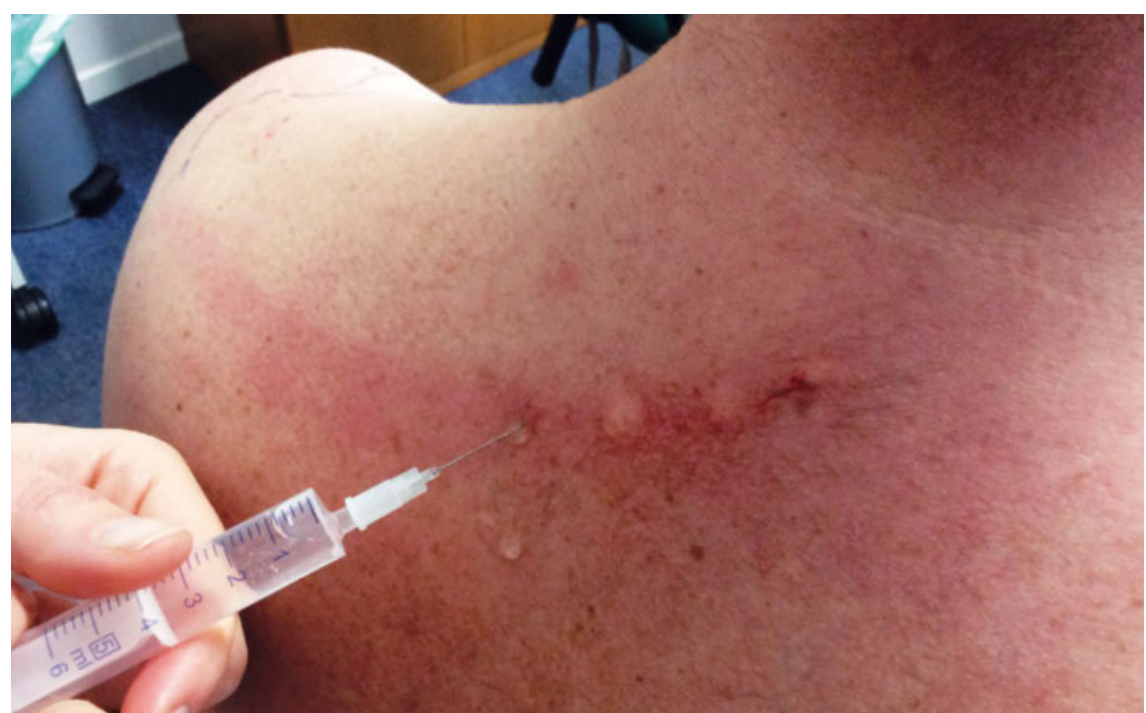

Abb. 2 Quaddeltechnik (intrakutane Infiltration) mit Procain. ten bei Erstluxationen in mehr als 40\% und bei Rezidiv-Luxationen in bis zu 90\% der Fälle auf [1]. Bei Instabilitäten ist das Akromioklavikulargelenk (ACG) zu 1/3 beteiligt [1].

Liegt eine Degeneration vor, werden bei 5-11\% der 50-Jährigen sowie bei bis zu 50\% der 70-Jährigen Rupturen der Rotatorenmanschette diagnostiziert [2].

\section{Differenzialdiagnostik der sog. Periarthropathie}

Die durch Duplay 1872 erstmals beschriebenen Veränderungen bei einer Periarthropathie lassen sich aktuell in raumfordernde,

\section{Zusammenfassung}

Die Neuraltherapie bildet eine gute Option die Beschwerden einer Omalgie zu lindern. Sie setzt sich aus lokaler bzw. Segmenttherapie, der erweiterten Segmenttherapie der Facettenregionen C3/4, C 4/5 und kaudaler sowie Injektionen an das Ganglion stellatum, ggf. in Kombination mit nervalen Strukturen und perivaskulärem Sympathikus, zusammen.

Bei anamnestischem Hinweis auf Phrenicusreizung sind der Plexus coeliacus in Verbindung mit Akupunktur und Homöosiniatrie je nach Organdiagnostik sowie die Störfeldtherapie je nach Blockierungsmuster und Nackenreflexpunkten nach Adler und Langer durchzuführen.

Bei Persistenz der o.g. Punkte stehen die stomatologische Diagnostik sowie Sanierungstherapie unter Nutzung von Ozon im Vordergrund.

Als Begleittherapie fungieren Manualtherapie, Antiphlogose, Homöopathie, autologes konditioniertes Plasma extraartikulär, Ozon intraartikulär, Procain-Basen-Infusionen bzw. intravenöse Oxygenierung sowie orthomolekulare Medizin. verkalkende und adhäsive Entzündungen zusammenfassen. Die Daten zu Synovialitiden sind unklar. Eigene Daten ergeben eine Häufigkeit von 22,9\% aller sonographisch untersuchten Synovialitiden der großen Gelenke.

In der Literatur wird bei Rupturen in mehr als 50\% der Fälle eine Tendinitis der langen Bizepssehne angegeben [3]. Verkalkungen der Insertionen treten meist im 30. bis 50. Lebensjahr mit einer Prävalenz von 3-20\% auf [4]. Eine frozen shoulder tritt gehäuft bei Diabetikern (bis zu 30\%) auf [5].

\section{Pathogenese aus neurovegata- tiver Sicht}

Dem Schmerz geht eine Entzündung voraus. Auch kurz nach einem Trauma treten v.a. in der Synovia vermehrt perivaskuläre Sympathikusfasern, die sensible Afferenzen modulieren, auf. Bei allen Degenerationen kommt es zusätzlich zur Reduzierung der Gefäße mit einer konsekutiven Hypooxygenierung [6].

Innerhalb eines jeden Segments reagieren Gefäße, Muskeln und Gelenkkomplexe. Afferente Reize im Bereich des N. phrenicus projizieren sich in die Dermatome C4, C3 und/oder C5. Andere Reize können bei Beteiligung der Ncll. Intermediolaterales in Höhe Th 3-6 den perivaskulären Sympathikus triggern, welcher die gesamte SchulterArm-Region versorgt [7]. Sogenannte neuromodulative Trigger bzw. Störfelder wirken über trigeminozervicale (v.a. dentogene, sinusogene und tonsillogene Entzündungen), aber auch über vagale und lumbosak- 


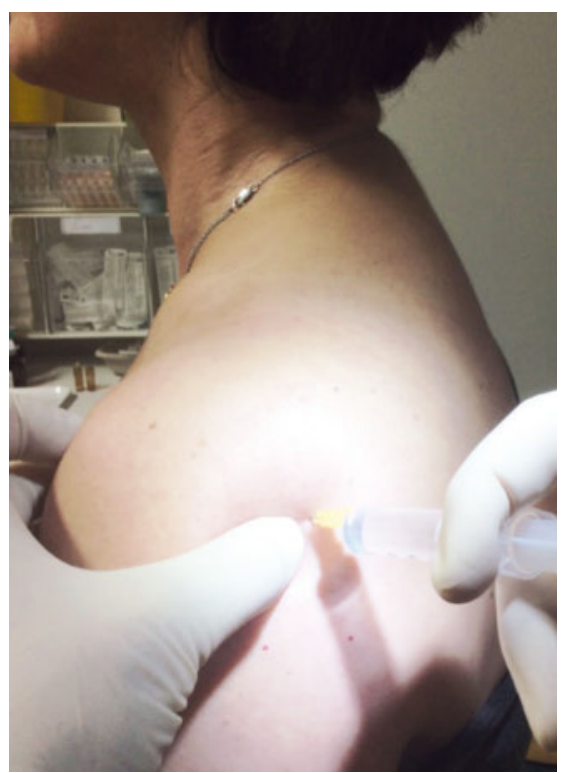

Abb. 3 Intraartikuläre Injektion in das glenohumerale Hauptgelenk links von dorsal.

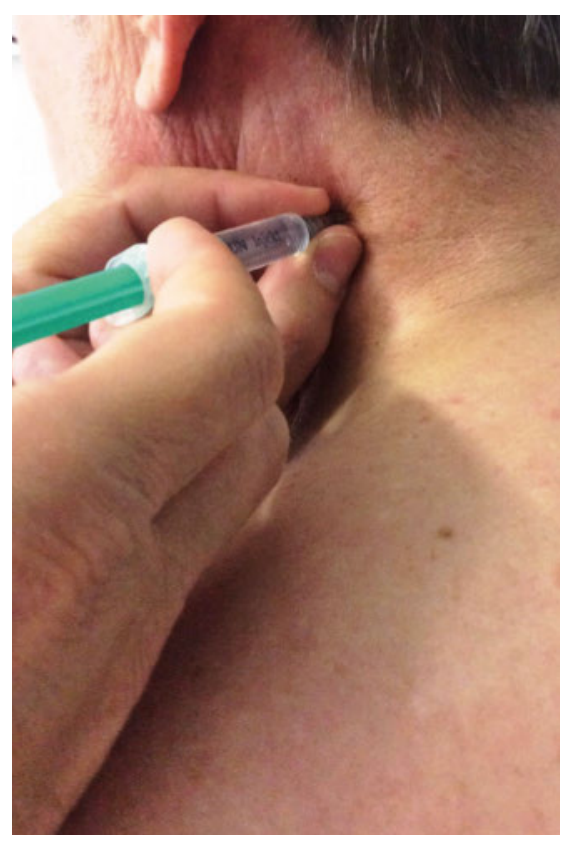

Abb. 5 Injektion an den N. accessorius links.

rale Interneurone (abdominelle, pelvine und retroperitoneale Reizzustände) [8].

\section{Symptomatik und Klinik}

Ein unterschiedlicher Verlauf zeichnet sich aus durch:

- eine functio läsa mit oder ohne einen painful arc,

- ein pathologisches Kapselmuster des glenohumeralen Hauptgelenks (GHG)

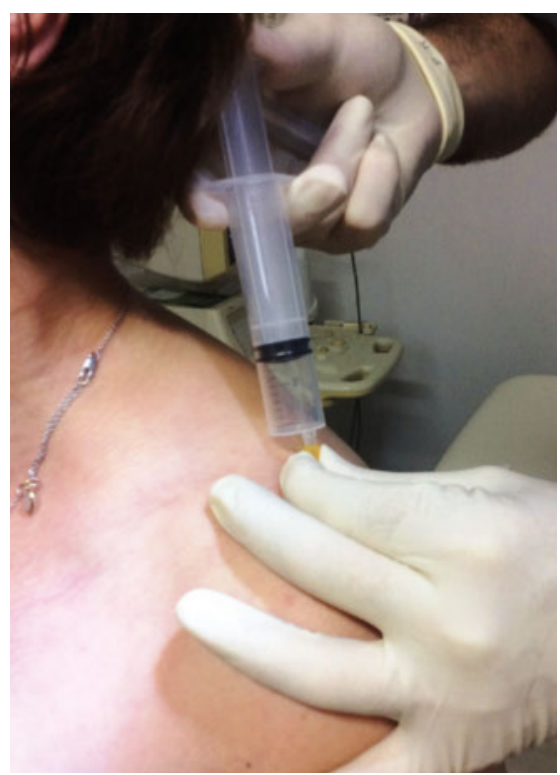

Abb. 4 Injektion von Ozon in die Bursa links von kranial.

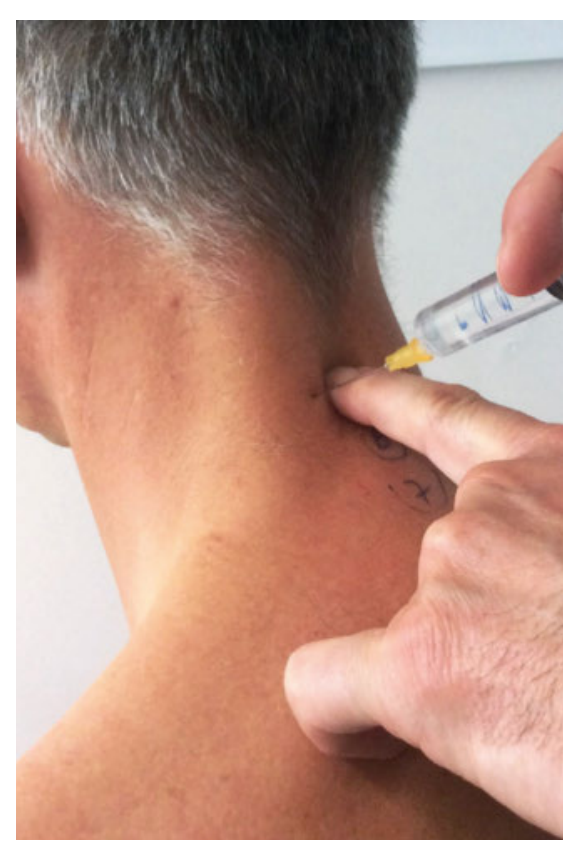

Abb. 6 Injektion an die Facettenregion nach Mink C4-5 links.

mit eingeschränkter Außenrotation sowie

- diverse Druck- und Triggerpunkte mit oder ohne einen referred pain.

Bekannte Zeichen an Sehnen und Kapseln nach Dugas, Neer, Hawkins, Speed, O’Brien usw. sowie an Gefäßen nach Adson, Falconer und Wright helfen bei der klinischen Diagnostik.
Der neuralgische Projektionsschmerz nach Head ist eine Ausschlussdiagnose!

Schulmedizinische Optionen sind Indikationen für operative Therapien und symptomatische Maßnahmen ohne klaren Indikationskatalog, welche dann ausgeschöpft werden sollten. $\mathrm{Zu}$ oft werden nicht-steroidale Antirheumatika und lokale Injektionen von Kortisonpräparaten mit erheblichem Risiko angewendet. Die physikalische Therapie ist teilweise zu unspezifisch in ihrer Indikation und Dokumentation, obwohl sie adjuvant bei nichtentzündlicher muskulärer Dysbalance notwendig ist.

Eine kortisonfreie antiinflammatorische Therapieoption besteht posttraumatisch, prä- sowie postoperativ immer in der Neuraltherapie.

\section{Neuraltherapie \\ Segmenttherapie}

Beim anamnestischen bzw. Spontanschmerz hilft eine Quaddel im Segment C4, welche auch auf die Segmente C5-Th 2 erweitert werden kann (Abb.2). Hierzu wird neben Procain auch Mepivacain empfohlen. Eine subkutane Injektion mit Procain kann, je nach anamnestischen und klinischen Hinweisen, auch durch Ozon oder Homöopathika ergänzt werden.

Je nach positivem Zeichen nach Dugas erfolgt die Injektion an/in das Acromioclaviculargelenk sowie an das Sternoklavikulargelenk.

Bei positivem Kapselmuster kann eine Injektion in das glenohumerale Hauptgelenk von dorsal (Abb.3) mit einer Injektion in die Bursa sowie Nutzung von Ozon und / oder Homöopathika kombiniert werden (Abb.4). Gezielte Injektion an dolente bzw. entzündete Muskeln und Sehnen können ebenfalls mit anderen Präparaten und einer Eigennosode aus dem Patientenserum ergänzt werden.

\section{Regionale Neuraltherapie}

Bei mono- oder oligosegmentaler Projektion, Persistenz bzw. Rezidiv der Beschwerden ist die Injektion an den $\mathrm{N}$. accessorius indiziert (Abb.5). Hier kann auch die Injektion an den N. supra-scapularis [9], an den Pl. brachialis [10] und/oder N. auricularis magnus helfen. Die Injektion an die Facettengelenke bzw. Rami articularis nach Mink sollte nach entsprechender Ausbildung, Aufklärung, Vor- und Nachbereitung an bis zu 3 Etagen zwischen C 4 und Th 6 (Abb.6) erfol- 


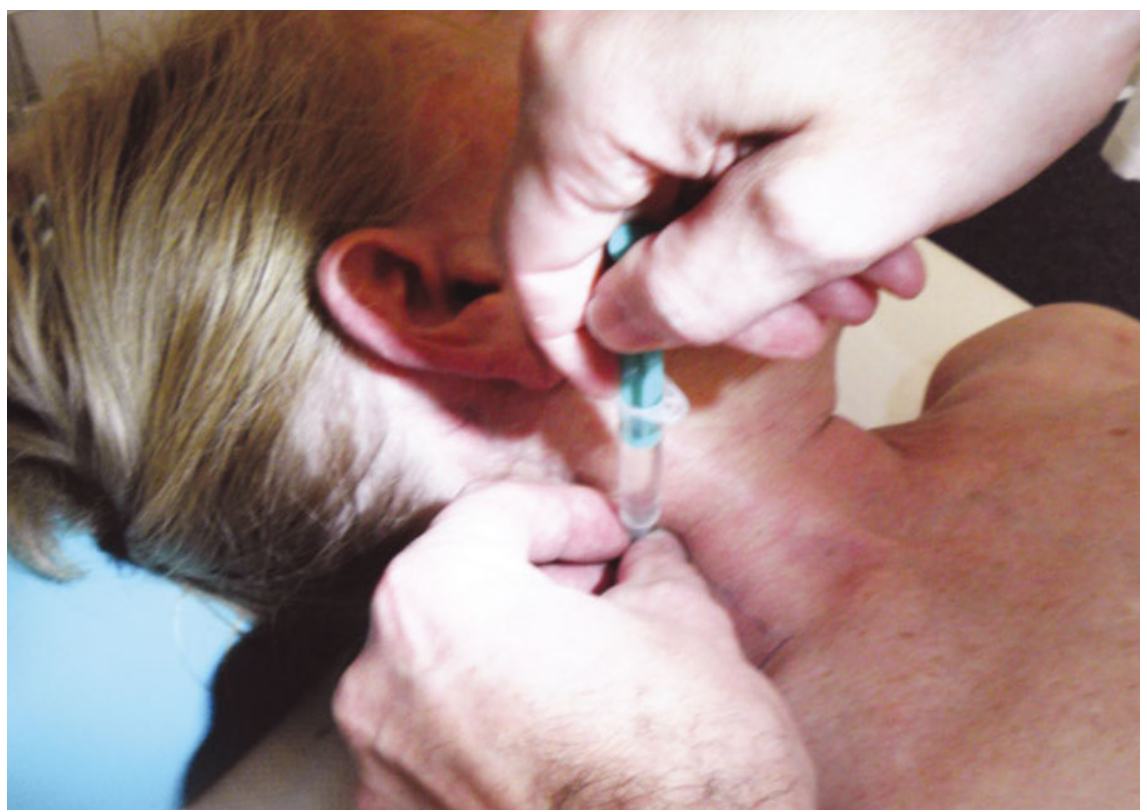

Abb. 7 Injektion an das Ganglion stellatum rechts.

gen. Sie hat wie die Injektion an das Ggl. stellatum (Abb. 7) die größte klinische Wirksamkeit und muss selten bzw. bei komplexen regionalen Schmerzsyndromen durch die Injektion an / in die A. axillaris sowie periphere Gefäße ergänzt werden.

\section{Neuraltherapie der neuromodulativen Trigger}

Die submuköse Injektion in das Velum palatinum (an die oberen und unteren Tonsillenpole) zeigen eine Wirkung u.a. auf die nuchale und die vom $\mathrm{N}$. accessorius versorg- te Muskulatur [11]. Sie können je nach Dolenz der Nackenreflexpunkte nach Adler und Langer [12, 13] durch die Injektion an die Endäste der Nn. trigemini 1 und 2 ( $\mathrm{Nn}$. supra- et infraorbitalis) sowie je nach Orthopantomogramm an die Odontone [14] ergänzt werden. Hier offenbaren sich häufig serologisch oder röntgenologisch erkennbare Entzündungen, die saniert werden müssen.

\section{Weitere adjuvante Verfahren}

Neben den o.g. Injektionen von Ozon [15] sind die Mesotherapie (z. B. bei der Tendinosis calcarea mit EDTA [16]), die Homöosiniatrie (z.B. mit Traumeel $\left.{ }^{\circledR}[17]\right)$, die Injektion von Eigenserum (z.B. mit Orthokin ${ }^{\circledR}[18]$ ), aber auch die Akupunktur, das sog. Dryneedling $[19,20]$ und das Kinesio-Taping [21] in verschiedenen Studien erfolgreich bewertet worden und in der täglichen Praxis empfehlenswert.

\section{Fazit für die Praxis}

Die Neuraltherapie kann komplementär zu konservativen und operativen Therapien eingesetzt werden. Sie wirkt antientzündlich und damit nicht nur schmerzlindernd, sondern über die Vasodilatation und Sym- 
pathikolyse regenerativ. Zudem ist sie zeitund kostensparend. Sie kann jederzeit mit anderen adjuvanten Verfahren der Naturheilkunde wie Akupunktur, Kinesio-Taping, Ozon-Therapie, Homöosiniatrie und Injektion von konditioniertem autologem Plasma kombiniert werden. Sie wird aktuell als eine der am häufigsten ambulant angewendeten Therapien angesehen und hat eine sehr niedrige Morbiditätsrate.

Interessenkonflikt: Der Autor erklärt, dass keine wirtschaftlichen oder persönlichen Verbindungen bestehen.

\section{Online zu finden unter}

http://dx.doi.org/10.1055/s-0043-106805

\section{Literatur}

$\overline{1}$ Doyscher R, Kraus K, Finke B et al. Akutverletzungen und Überlastungsschäden der Schulter im Sport. Der Orthopäde 2014; 3: 202-207

$\overline{2}$ Habermeyer P, Lehmann L, Lichtenberg S. Rotatorenmanschetten-Ruptur. Orthopäde 2000; 29 : 196-208

$\overline{3}$ Murthi AM et al. The incidence of pathologic changes of the long head of the biceps tendon. J Shoulder Elbow Surg 2000; 9 (5):382-385

$\overline{4}$ Magosch P, Lichtenberg S, Habermeyer P. Effizienz der Radialen Stosswellentherapie bei der Tendinosis calcarea der Rotatorenmanschette - Eine prospektive Studie. 2004; Im Internet: www.enimed.dk/pdf/documentation/de/ Buch07.pdf

5 Hertel R. Die steife Schulter. Orthopäde 2000; 29: 845-851

$\overline{6}$ Peuker ET. Neuroanatomische Grundlagen des Gelenkschmerzes. Akt Rheumatol 2016; 41: 300-305

$\overline{7}$ Wancura-Kampik I. Segment-Anatomie. 2. Aufl. München / Jena: Urban \& Fischer; 2010

$\overline{8}$ Saha FJ, Wander R. Das Störfeld als neuromodulativer Trigger auf allen Ebenen. DZA 2014; 57 : 6-9

9 Jeske HC et al. A randomized study of the effectiveness of suprascapular nerve block in patient satisfaction and outcome after arthroscopic subacromial decompression. Arthroscopy 2011; 27 (10): 1323-1328

$\overline{10}$ Jafari S, Kalstein AI, Nasrullah HM et al. A randomized, prospective, double-blind trial comparing $3 \%$ chloroprocaine followed by $0.5 \%$ bupivacaine to $2 \%$ lidocaine followed by $0.5 \%$ bupivacaine for interscalene brachial plexus block. Anaesth Analg 2008; 107 (5): 1746-1750

$\overline{11}$ Weinschenk S, Hollmann MW, Göllner R et al. Injections of local anesthetics into the pharyngeal region reduce trapezius muscle tenderness. Forsch Komplementmed 2016; 23 (2): 111-116

$\overline{12}$ Adler E. Erkrankungen durch Störfelder im Trigeminusbereich. Heidelberg: Fischer; 1973

$\overline{13}$ Langer H. Die Adler'schen Druckpunkte, Ursachen und Problematik. Vortrag auf der 1. Arbeitstagung der AG Neuraltherapie. Holzhau: 1977

$\overline{14}$ Gleditsch JM. Mundakupunktur. Schorndorf: WBV; 1979

$\overline{15}$ Chen H, Yu B, Lu C et al. The effect of intra-articular injection of different concentrations of ozone on the level of TNF- $\alpha$, TNF-R1, and TNF-R2 in rats with rheumatoid arthritis. Rheumatol Int 2013; 33 (5): 1223-1227

$\overline{16}$ Soncini G, Costantino $\mathrm{C}$. The treatment of pathologic calcification of shoulder tendons with E.D.T.A. bisodium salt by mesotherapy. Acta Biomed 1998; 69 (5-6): 133-138

$\overline{17}$ Vanden Bossche L, Vanderstraeten G. A multicenter, double-blind, randomized, placebo-controlled trial protocol to assess Traumeel injection vs dexamethasone injection in rotator cuff syndrome: the Traumeel in Rotator cuff syndrome (TRARO) study protocol. BMC Musculoskelet Disord 2015; doi: 10.1186/s12891-015-0471-z

$\overline{18}$ Auw Yang KG, Raijmakers NJ, van Arkel ER et al. Autologous interleukin-1 receptor antagonist improves function and symptoms in osteoarthritis when compared to placebo in a prospective randomized controlled trial. Osteoarthritis Cartilage 2008; 16 (4): 498-505

$\overline{19}$ Hidalgo-Lozano A, Fernandez-de-las-Penas C, Diaz-Rodriguez $\mathrm{L}$ et al. Changes in pain and pressure pain sensitivity after manual treatment of active trigger points in patients with unilateral shoulder impingement: a case series. J Bodyw Mov Ther 2011; 15 (4): 399-404

$\overline{20}$ Koppenhaver S, Embry R, Ciccarello J et al. Effects of dry needling to the symptomatic versus control shoulder in patients with unilateral subacromial pain syndrome. Man Ther 2016; 26: 62-69

$\overline{21}$ Kalter J et al. Taping patients with clinical signs of subacromial impingement syndrome: the design of a randomized controlled trial. BMC Musculoskelet disord 2011; 12: 188

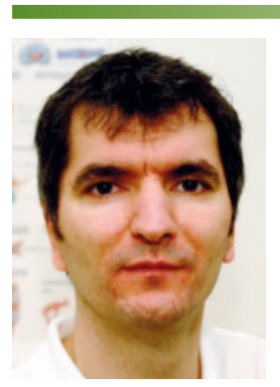

\section{Dr. med. Uwe Günter}

Siegfriedstr. 204c

10365 Berlin

info@dr-guenter.de

www.biologische-orthopädie-berlin.de

Uwe Günter ist Facharzt für Orthopädie und Unfallchirurgie mit den Zusatzbezeichnungen Chirotherapie, Akupunktur, Sportmedizin und Physikalische Therapie. Niederlassung in eigener Praxis mit Schwerpunkt Neuraltherapie in Berlin. Dozent der Deutschen Gesellschaft für Akupunktur und Neuraltherapie. 